\title{
Effects of resonant magnetic perturbations on turbulence and transport in DIII-D L-mode plasmas
}

\author{
T. L. Rhodes \\ L. Zeng \\ E. J. Doyle \\ et al.
}

Saskia Mordijck

William \& Mary, smordijck@wm.edu

Follow this and additional works at: https://scholarworks.wm.edu/aspubs

Part of the Physics Commons

\section{Recommended Citation}

Mordijck, S., Rhodes, T. L., Zeng, L., Doyle, E. J., Schmitz, L., Chrystal, C., ... \& Moyer, R. A. (2015). Effects of resonant magnetic perturbations on turbulence and transport in DIII-D L-mode plasmas. Plasma Physics and Controlled Fusion, 58(1), 014003.

This Article is brought to you for free and open access by the Arts and Sciences at W\&M ScholarWorks. It has been accepted for inclusion in Arts \& Sciences Articles by an authorized administrator of W\&M ScholarWorks. For more information, please contact scholarworks@wm.edu. 


\title{
Effects of resonant magnetic perturbations on turbulence and transport in DIII-D L-mode plasmas
}

\author{
S Mordijck ${ }^{1}$, T L Rhodes ${ }^{2}$, L Zeng ${ }^{2}$, E J Doyle ${ }^{2}$, L Schmitz ${ }^{2}$, C Chrystal ${ }^{3}$, \\ T J Strait ${ }^{4}$ and R A Moyer ${ }^{5}$ \\ 1 Department of Computer Science, College of William and Mary, Williamsburg, VA 23187, USA \\ 2 Department of Physics and Astronomy, University of California Los Angeles, Los Angeles, CA 90095, \\ USA \\ 3 ORAU, 5 Le Way Dr, Fredericksburg, VA 22406, USA \\ ${ }^{4}$ General Atomics, PO BOX 85608, San Diego, CA 92186-5608, USA \\ 5 Center for Energy Research, University of California San Diego, 9500 Gilman Dr., La Jolla, \\ CA 92093-0417, USA \\ E-mail: mordijck@cs.wm.edu
}

Received 1 July 2015, revised 21 August 2015

Accepted for publication 7 September 2015

Published 20 October 2015

\begin{abstract}
In this paper we show that resonant magnetic perturbations (RMPs) affect the L- to H-mode power threshold. We find that during the L-mode phase, RMPs cause the particle pinch to reverse from traditionally inward to outward. As a result, the density at the plasma edge increases, while the density in the plasma core is reduced. Linear stability calculations indicate that the plasma transitions from an ion temperature gradient (ITG) to trapped electron mode (TEM) regime at the plasma edge. If the applied RMP current is below the threshold for penetration and island formation, we find that the changes in the edge radial electric field are minimal, while the carbon toroidal rotation brakes over the whole minor radius. Once the RMP field penetrates and the screening plasma response dissappears, the spin-up of the toroidal rotation at the plasma edge results in a positive radial electric field inside the separatrix.
\end{abstract}

Keywords: tokamaks, turbulence, transport, RMPs

(Some figures may appear in colour only in the online journal)

\section{Introduction}

The use of resonant magnetic perturbations (RMPs) in tokamaks has been demonstrated to be a promising technique to suppress and mitigate edge localized modes (ELMs) along with providing an extra control knob to regulate confinement $[1,2]$. Therefore, future tokamak devices, such as ITER, are relying predominantly on RMPs to mitigate or suppress ELMs, to reduce the intermittent high heat fluxes to the divertor [3]. While in current devices the heat fluxes associated from large ELMs can damage metallic tiles, they do not destroy the divertor and limit operations [4]. However, the extrapolated power loss per ELM in ITER will result in serious damage to the first wall [5]. As a result, the first ELM needs to be avoided on ITER. ELMs are a distinct feature of standard $\mathrm{H}$-mode confinement [6] and so the best strategy to avoid the first ELM in ITER is to apply RMPs during the L-mode phase, before the plasma transitions from L- to H-mode.

Previous research studying the effects of RMPs on the L- to $\mathrm{H}$-mode power threshold has shown that RMPs can increase the power threshold $\left(P_{\mathrm{L}-\mathrm{H}}\right)$ for the $\mathrm{L}$ - to $\mathrm{H}$-mode power transition [7-10]. Our current understanding of the dynamics affecting the L- to $\mathrm{H}$-mode transition rely upon the interaction between turbulence (through Zonal Flows) and build up of an equilibrium shear layer in H-mode [11]. So in order to better understand how RMPs affect the power threshold, we need to study the influence of RMPs upon turbulence, transport and shear flow in L-mode plasmas, well before the L- to H-mode 
transition. While studying the effects of RMPs upon the power threshold in MAST, depending on the applied spectrum (and above a minimum RMP strength) an increase of 20-60\% in the power threshold was observed [10]. In DIII-D an increase of up to $100 \%$ was observed when the RMPs were applied within the 'resonant window' for ELM suppression at typical coil current values needed for suppression [7]. In AUG experiments, an increase of $20 \%$ in the $P_{\mathrm{L}-\mathrm{H}}$ was observed [8]. In these discharges, the transition to H-mode with RMPs is observed to occur at higher, less negative radial electric field. In NSTX an increase in $P_{\mathrm{L}-\mathrm{H}}$ of $50 \%$ is observed [9].

Most of the work with respect to the effect of RMPs upon transport in L-mode plasmas was performed in limiter devices such as TEXTOR and TORE-SUPRA [12-15]. In these plasmas, depending on the RMP spectrum, either an improvement or a decrease in confinement could be observed. The specific changes in the electron density and the electron temperature gradient depend strongly on the exact 3D magnetic topology [14]. Typically, a distinction is made between the ergodic region and the laminar region. The difference between the two regions is whether the length of the fields lines is longer (ergodic) or shorter (laminar) than the Kolmogorov length, where the Kolmogorov length defines the decorrelation length between two neighboring field lines in the stochastic region. In the laminar region, the electron temperature is flat, whereas in the ergodic region there is nearly no change in the electron temperature gradient. The electron density also flattens in the laminar region, but steepens in the ergodic region. At the same time, probe measurements show a reversal of the particle flux in the ergodic region, from outward without RMPs to inward with RMPs [15]. The largest change can be observed in the radial electric field, $E_{\mathrm{r}}$. Outside the LCFS the $E_{\mathrm{r}}$ becomes positive whereas in the laminar zone, a strong reduction in $E_{\mathrm{r}}$ results in the creation of a small well. The magnetic topology also affects turbulence measurements; in the ergodic region, a strong reduction in large scale structures is observed as well as a reduction in the poloidal and radial correlation lengths for all frequencies [15]. In the laminar zone, the changes in equilibrium shear dominate the changes in turbulence. In the ergodic zone, previously coherent modes that are observed in ohmic plasmas are suppressed and frequency spectra become more exponential. The Reynolds stress is fully quenched by the RMPs in TEXTOR in the laminar region [16]. In MAST, a diverted spherical tokamak, no increase in confinement in L-mode plasmas was observed, independent of the applied spectrum. In MAST, an increase in $E_{\mathrm{r}}$ was observed, once the RMP current was above a threshold value. Concurrently with the changes in $E_{\mathrm{r}}$ a reduction in density and an increase in fluctuations were observed [17].

In this paper we show that RMPs increase the $P_{\mathrm{L}-\mathrm{H}}$ in DIII-D from 2.9 MW to 3.3 MW in the case of a zero torque injected, neutral beam (NBI) heated plasma in the ITER similar shape (ISS) when the coil current is $4 \mathrm{kA}$ for an even parity $n=3$ spectrum, with $n$ being the toroidal mode number configuration, within the 'resonant window' for ELM suppression (see section 2). We find that just before the $P_{\mathrm{L}-\mathrm{H}}$ transition, the edge ion temperature is about a factor 2 higher for the RMP discharge in comparison to the non-RMP case (due to the higher NBI input power) and there is a strong shear in the edge carbon toroidal rotation, which is not observed in the non-RMP discharge.

In a set of dedicated experiments to study the effects of RMPs upon turbulence and transport in L-mode plasmas in DIII-D, we find that a reduction in confinement can be observed above a threshold in RMP strength (see section 3). The reduction in confinement coincides with the disappearance of the plasma response and the locking of the core rotation at the $q=3 / 2$ surface. Below this threshold we do observe a small reduction in the core density, which is countered by a small increase in the edge density. We find that at the plasma edge, the turbulent flow measured by the DBS reverses in the lab frame. Similarly, quasi-linear TGLF simulations show a transition from an ITG to TEM dominant regime. Along with these changes in turbulence, we also observe a strong change in the measured perturbed inward pinch. In the non-RMP discharge, which is in the ITG regime the pinch is inward, whereas with RMPs the pinch changes to outward along with a change in linear stability to the TEM regime at the plasma edge. This is the first direct measurement which shows that the particle pinch changes direction when the turbulence regime changes from ITG to TEM, a well known theoretical phenomenon [18]. Prior experimental results on transitioning from ITG to TEM regimes, showed a local increase in the density gradient, which acts as a proxy for a change in the v/D ratio [19]. Finally, in section 4, we will discuss these results and how they might affect predictions for ITER.

\section{Increase in L- to $\mathrm{H}$ power threshold}

Previous experiments have shown that RMPs increase the L- to H-mode power threshold in DIII-D [7]. The cause for this increase in $P_{\mathrm{L}-\mathrm{H}}$ is not yet understood. A more careful look at the experiments that were performed to study the L-H transition with RMPs inside the 'resonant window' with $q_{95} \sim 3.6$ for ELM suppression shows that RMPs have little effect upon the line averaged density, see figure 1 where black is the non-RMP discharge (141991) and red is the RMP discharge (141992). These plasmas have balanced NBI injection and the core $(\rho \sim 0.15)$ toroidal carbon rotation is slightly lower in the discharge with the RMPs, however rotation at the plasma edge $(\rho \sim 0.85)$ is similar for both discharges. We can also observe that the first ELM is not suppressed, which is most likely the result of the balanced torque injection. So far, DIII-D has not been able to suppress ELMs in low or balanced torque injected discharges with RMPs at high $\beta$.

Electron density and temperature profile data is unavailable for the discharge with RMPs. So we are limited to comparing the changes in ion temperature and toroidal rotation. Recent work on the dynamics of the L- to H-transition has shown that the ion temperature gradient is an important driver in accessing $\mathrm{H}$-mode [20]. The ion temperature in the RMP discharge is twice that of the non-RMP discharge (see figure 2(a)), as a result of the increase in NBI heating (the small increase at the edge should be ignored and is an artifact). On the other hand, the carbon toroidal rotation profile shows a steep rotational well in the RMP discharge at the plasma edge, see figure 2(b). 


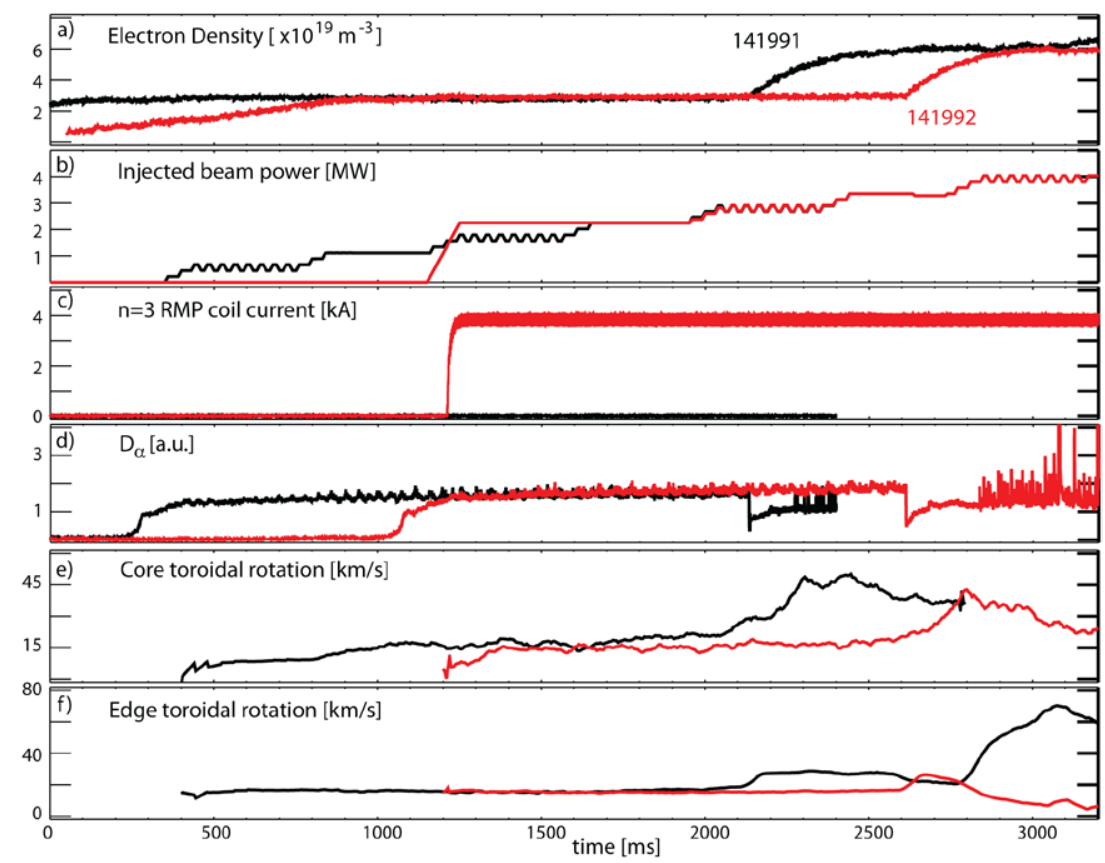

Figure 1. Time evolution of two discharges in which the NBI power was stepwise increased (b) to the $\mathrm{L}$ to $\mathrm{H}$ power threshold changes (a) as can be seen in the sudden line averaged density increase with the application of RMPs (c). The time traces for the discharge without RMPs were shifted by $500 \mathrm{~ms}$, to have overlapping power steps. The power threshold increases when RMPs are applied. The edge rotation was fairly similar for the RMP on and off discharges before the transition (f), while the core rotation is slightly reduced with RMPs (e).

As a result, the flow shear at the plasma edge is much larger in the plasma with RMPs. These changes at the plasma edge motivate us to study the effect of RMPs upon turbulence and transport at the plasma edge in L-mode plasmas in the next section.

\section{Effect of RMPs in L-mode plasmas}

\subsection{Experimental setup}

In this paper, we introduce a set of three L-mode discharges in the ISS shape in which the amount of RMP (as defined by the coil current) was varied from no RMP (black, discharge 142613 ), to $5.0 \pm 0.2 \mathrm{kA}$ (red, discharge 142617 ) and finally $5.6 \pm 0.2 \mathrm{kA}$ (blue, discharge 142618 ), see figure 3 . All three discharges were heated using $1 \mathrm{MW}$ of NBI heating power injecting $1 \mathrm{Nm}$ of co-torque. The toroidal magnetic field, $B_{\mathrm{T}}$, is $2.0 \mathrm{~T}$ and the plasma current, $I_{p}$ is $1.4 \mathrm{MA}$, which results in a $q_{95}$ (safety factor at the $95 \%$ flux surface) of 3.6. The line averaged density is $3.8 \times 10^{19-3}$ (before the application of RMPs) and a collisionality of $\nu^{*}=q_{95} R \epsilon^{-3 / 2} \lambda_{e}^{-1} \sim 10$. Here, $R$ is the major radius, $\epsilon(\equiv a / R)$ is the inverse aspect ratio, $a$ is the minor radius and $\lambda=v_{T_{e}} \tau_{e}$, the product of electron thermal velocity $v_{T_{\mathrm{e}}}$ and electron-electron collision time $\tau_{e}$. All three discharges have the same level of gas fueling. The $n=3$ even parity RMPs are activated at $1630 \mathrm{~ms}$, see figure 3 . There is initially no change in the line averaged density, however at $2000 \mathrm{~ms}$, the discharge with the higher RMP coil current shows a decrease in the density. This occurs simulateously with a strong sudden drop of the core rotation to about $5 \mathrm{~km} \mathrm{~s}^{-1}$ at the $q=3 / 2$ surface $(\rho \sim 0.61)$. This is an indication that a non-rotating island is created (similar to a locked mode, without the typical disruption). Once the RMPs are deactivated, the core plasma spins back up.

Next, we take a closer look at the experimental profiles and note that even though the line averaged density didn't change for the $5.0 \mathrm{kA}$ discharge, the core density dropped. This drop is offset by an increase in the edge and SOL density, see figure 4(a). For $5.6 \mathrm{kA}$, the core density drops further, but the edge density remain similar to the case with less coil current. The electron temperature does not change when 5.0 kA RMP current is applied, but at higher current, there is a reduction in the core electron temperature, see figure 4(b). At the plasma edge, the changes in electron temperature are within error bars. The core ion temperature is reduced with increasing RMP coil current, see figure 4(c). The carbon toroidal rotation breaks in the core plasma when RMPs are applied, see figure 4(d). For the $5.0 \mathrm{kA}$ discharge, the rotation slows over the whole minor radius. For the $5.6 \mathrm{kA}$ discharge, the rotation is close to zero between $\rho=0.6$ and $\rho=0.7$. There is a clear spin up of the rotation at the plasma edge, which is characteristic of the creation of a stochastic edge topology [21]. This observation is supported by observations of tangle splitting in the divertor area after the bifurcation of the toroidal rotation and the dissappearance of the $n=3$ plasma response [22].

\subsection{Plasma response}

In order to better understand how RMPs affect these plasmas, we need to examine the experimental plasma response. In these experiments we were able to measure the $n=1$ and the $n=3$ plasma response with the magnetic probes and coils at the vessel wall on the low field side, see figure 5. When the RMP coils are activated, there is a strong increase in the $n=3$ 

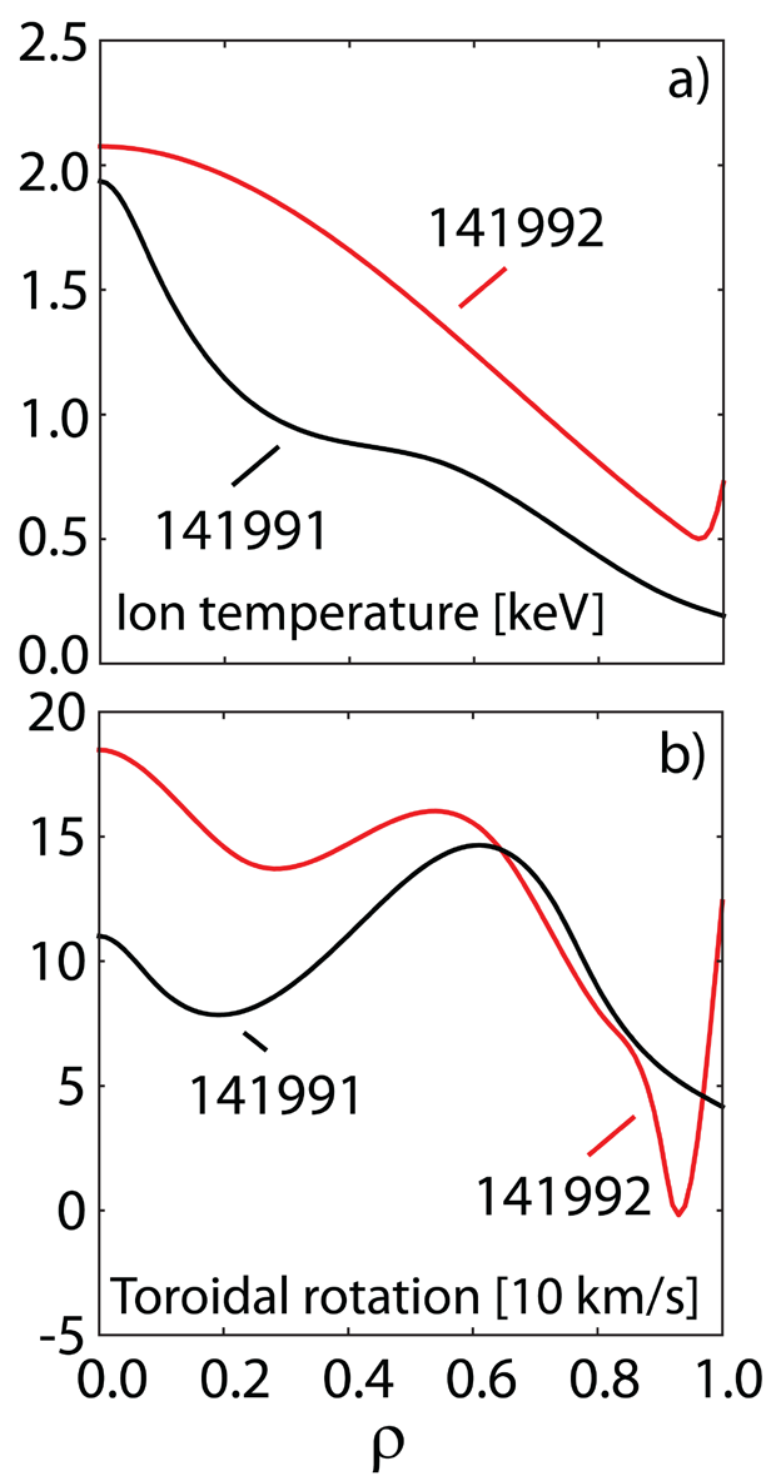

Figure 2. (a) Ion temperature and (b) carbon toroidal rotation just before the L- to H-mode transition for the non-RMP (black) and RMP (red) discharge. The higher NBI power results in a higher Ti profile for the RMP discharge. The core rotation is higher just before the transition (both balanced injected torque) for the RMP discharge. There is a strong shear layer in the toroidal rotation at the plasma edge, even before the transition.

plasma response. Although we apply the typical error-field correction in these plasmas there is also a strong increase in the $n=1$ plasma response. At the time when the core toroidal rotation collapses (see figure $4(\mathrm{~d})$ in the $5.6 \mathrm{kA}$ discharge, the $n=3$ plasma response is reduced to the levels of the $n=1$ plasma response. This reduction in the plasma response is not captured by linear single fluid MHD modeling [23].

That a linear single fluid MHD simulation would not capture the creation of an island along with a stochastic edge boundary shouldn't come as a surprise. In order for an island to form, the magnetic flux surfaces need to be compressed in order for reconnection to occur. These plasmas have regular sawteeth and the disappearance of the $n=3$ plasma response, along with the bifurcation of the toroidal rotation at the $q=3 / 2(\rho \sim 0.61)$ surface, occurs after such a sawteeth crash, see figure 6 . With the most rapid change in the toroidal rotation occurring at the $q=3 / 2$ surface, this indicates that this is the result of the $n=2$ error field penetrating, not the applied $n=3$ RMPs. The $n=3$ RMPs slow the core plasma down to the point where the $n=2$ error field can penetrate. The island strongly reduces the rotation over the whole radial profile, thus allowing the $n=3$ field to create a stochastic 3D edge.

The creation of the $3 \mathrm{D}$ tangles during this phase can be observed in the divertor [22]. Along with the spin up of the toroidal rotation at the plasma edge, this all points to the creation of an stochastic edge region. However, this 3D edge does not seem to affect the edge density profile, whereas the island seems to strongly reduce the electron density as well as the electron temperature in the plasma core. The electron temperature measured with the ECE is not flat (see figure 4(b)), but temperature flattening is only expected in the O-point of the island. However, the electron temperature profile using the Thomson scattering system, located at different poloidal and toroidal location than the ECE system, shows some flattening just outside $\rho=0.6$, see figure 7. Even without the creation of a 3D stochastic edge, RMPs can deform the separatrix into a complex 3D structure. In order to make sure that the 3D deformation of the separatrix is not the cause of the changes in the experimental profiles we compared the $2 \mathrm{D}$ separatrix values of multiple diagnostics. We did not observe a displacement of the separatrix. This is in agreement with recent observations in DIII-D H-mode plasmas, where the displacement of the separatrix with $n=3$ RMPs is minimal [24].

\subsection{Particle transport}

In order to study how particle transport is affected by RMPs in L-mode plasmas, we used a perturbative gas puff technique [25]. The application of a short gas puff (20 ms) every $200 \mathrm{~ms}$ perturbs the density profile at a constant frequency. The goal is to keep the perturbation small in order to minimize the effect on the electron temperature as well as minize the effect of the additional fuelling source. In these L-mode plasmas, this results in $20 \mathrm{~ms}$ of 160 Torr L gas injection every $200 \mathrm{~ms}$ to allow the density to relax back to its original steady-state value. Multiple repetitions of this perturbative puff allow us to Fourier decompose the perturbation into an amplitude and a phase. Next, linearizing the perturbed continuity equation allows us to express the diffusion coefficient and the pinch as a function of this amplitude and phase [26]. In figure 8, we note that during the RMP phase, there is a small increase in the diffusion coefficient, $D_{p}$. However, the large change is in the inward pinch, $v_{p}$. The inward pinch is strongly reduced at the plasma edge and close to the separatrix, where for the highest RMP current, the pinch reverses.

The mathematical technique to calculate $D_{p}$ and $v_{p}$ does not include the steady state source, nor the extra edge ionization source from the gas puff at the plasma edge [26]. This means we have to be careful when interpreting these results. In a sourceless region of the plasma, we can assume that $v_{p} / D_{p} \sim 1 / n \partial n / \partial r=1 / L_{n}$. In the core of these plasmas, we still have a small fueling source from the beams and so when 


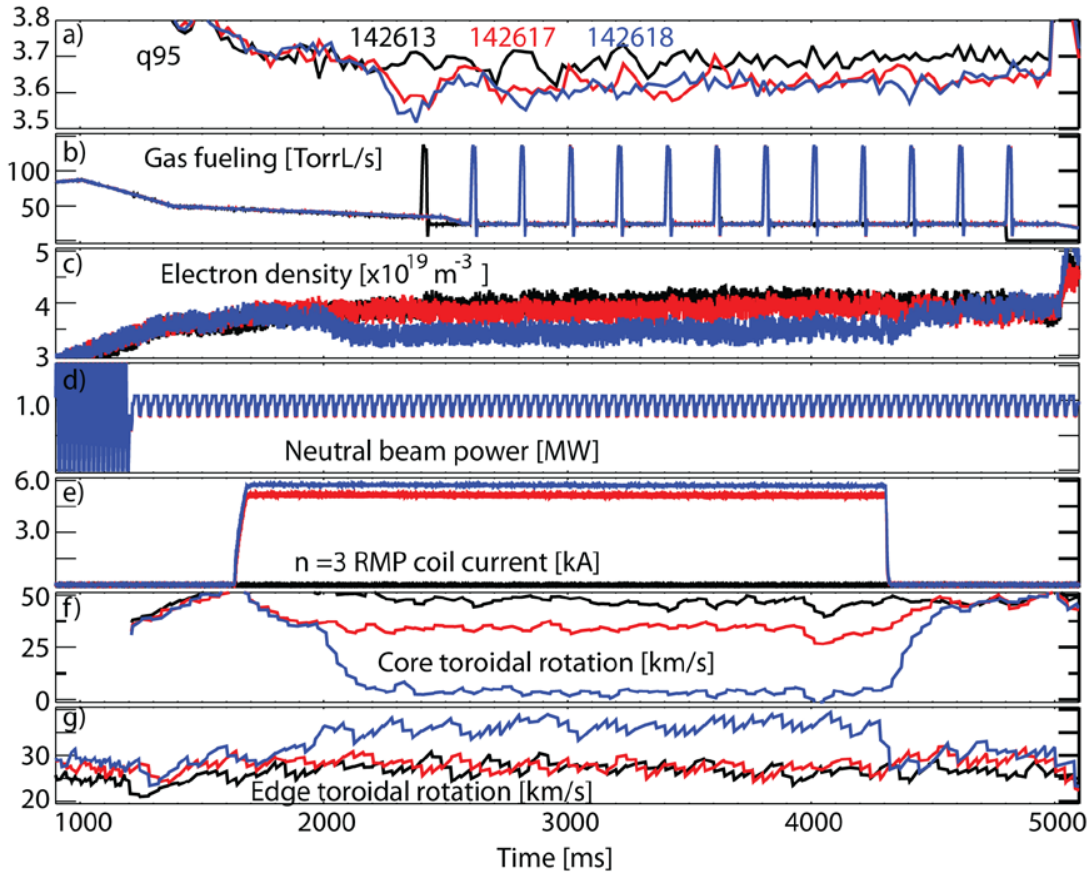

Figure 3. Time evolution of three L-mode discharges at similar $95 q$ (a), with similar fueling profiles and small perturbative gas puffs to measure the particle transport (b) where the level of RMPs is varied from none (black) to $5.0 \mathrm{kA}$ (red) and $5.6 \mathrm{kA}$ (blue) (e). When the RMPs are applied, there is a reduction in core rotation (f), while the edge rotation remains similar ( $\mathrm{g}$ ). In the case with the highest RMP current (blue), there is a sudden drop in core rotation along with a reduction in line averaged density at $2000 \mathrm{~ms}$ (c).
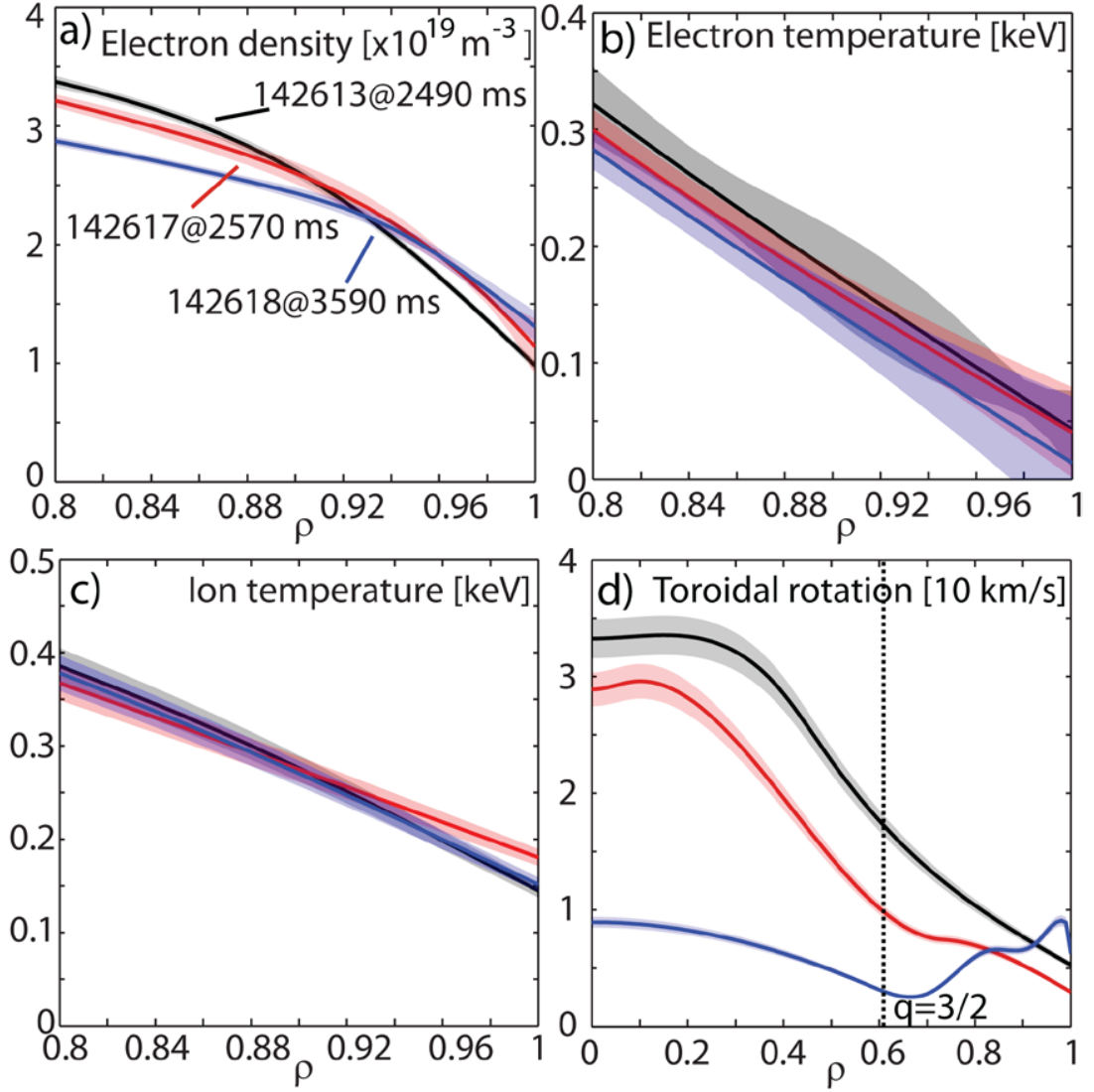

Figure 4. (a) Edge density profile measured with the reflectometer for the three discharges from figure 3 . There is a small increase in edge density, while the density drops in the core. (b) The edge electron temperature does not change much. (c) The edge ion temperature is similar for all the discharges. (d) The rotation drops over the whole radial profile for the $5.0 \mathrm{kA}$ (red) discharge and decreases further for the $5.6 \mathrm{kA}$ (blue) discharge in the core, whereas at the plasma edge, the rotation increases. 


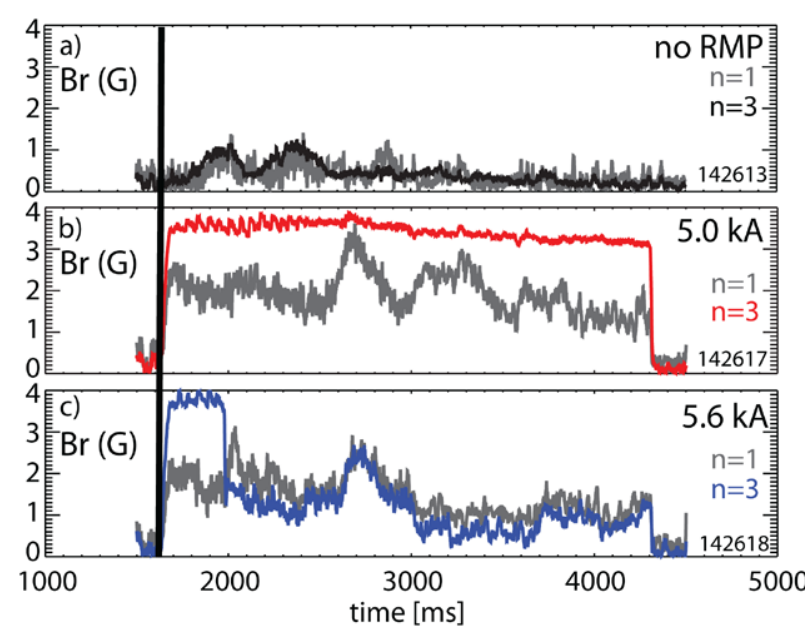

Figure 5. Time evolution of the $n=1$ and the $n=3$ plasma response for the three discharges from figure 3 . The black line is when the $n=3$ RMP coils are energized. (b) There is a strong increase in the $n=3$ response in the $5.0 \mathrm{kA}$ discharge. (c) For the $5.6 \mathrm{kA}$ discharge there is first a strong $n=3$ response, which then collapses close to $2000 \mathrm{~ms}$ to values similar to the $n=1$ response

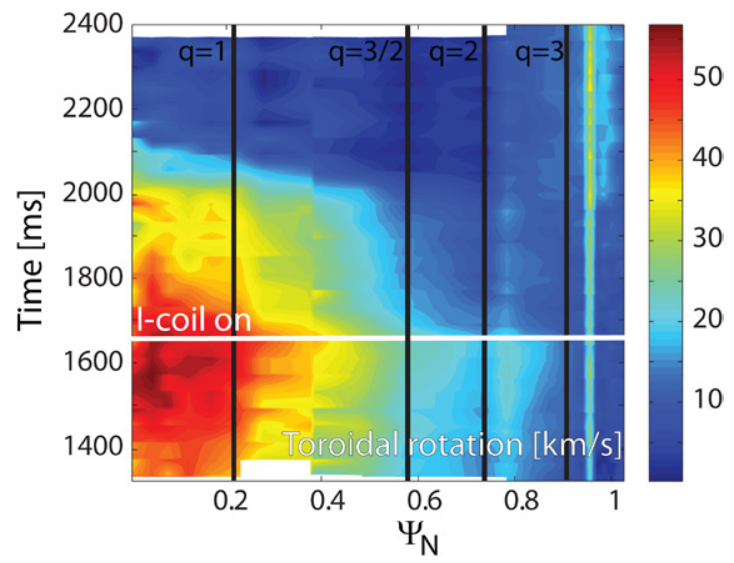

Figure 6. The evolution of the toroidal rotation versus radius and time for the $5.6 \mathrm{kA}$ discharge. The black vertical lines indicate the location of the rational surfaces. When the RMPs are applied, the rotation drops first close to the $q=2$ surface. The second collapse around $2000 \mathrm{~ms}$ occurs close to the $q=3 / 2$ rational surface.

comparing $v_{p} / D_{p}$ in the core with $1 / L_{n}$ this needs to be taken into account:

$$
v_{p} / D_{p}=1 / L_{n}+\frac{\int S}{D n}
$$

Currently, we do not have a good model in the 1D transport codes to calculate the ionization source at the plasma edge and we have therefore limited the comparison to the core plasma. In figure 9, we find that for discharges without RMPs and with $5.0 \mathrm{kA}$ that the $v_{p} / D_{p}$ coefficient predicts a density slope twice as steep as experimentally observed while $\left(\frac{\int S}{D n}\right.$ adds a fixed offset of about 0.1 to $1 / L_{n}$ ). The $v_{p} / D_{p}$ for the discharge where the $3 \mathrm{D}$ field penetrates (blue) predicts a nearly fixed density gradient, however, this is not observed experimentally. Previous work on how particle transport changes across an island on LHD showed that the density flattens inside

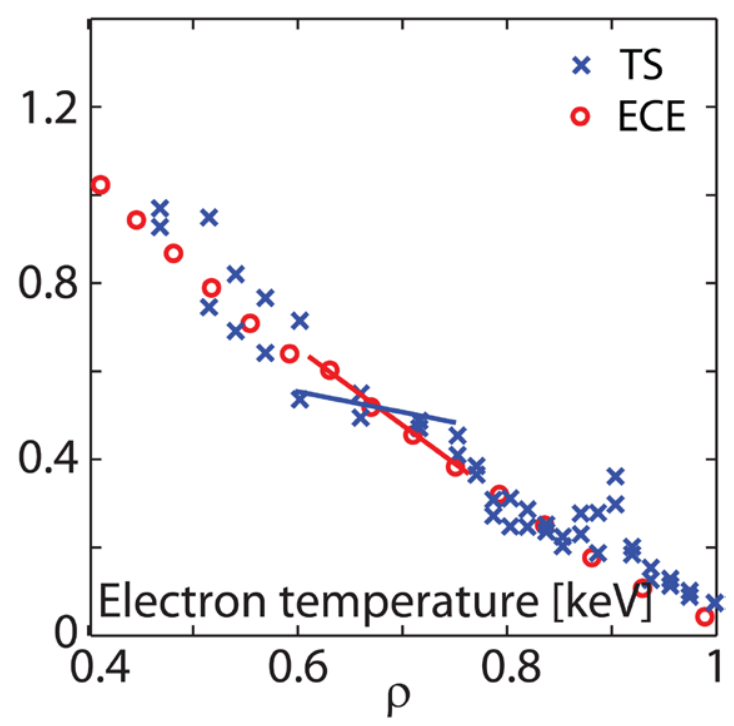

Figure 7. The electron temperature for discharge 142618 after the rotation collapse measured by the electron cyclotron emission (ECE, red) and the Thomson scattering (TS, blue). While no flattening of the electron temperature is observed with the ECE, the TS system shows flattening outside $\rho \sim 0.6$ and $\rho \sim 0.8$.

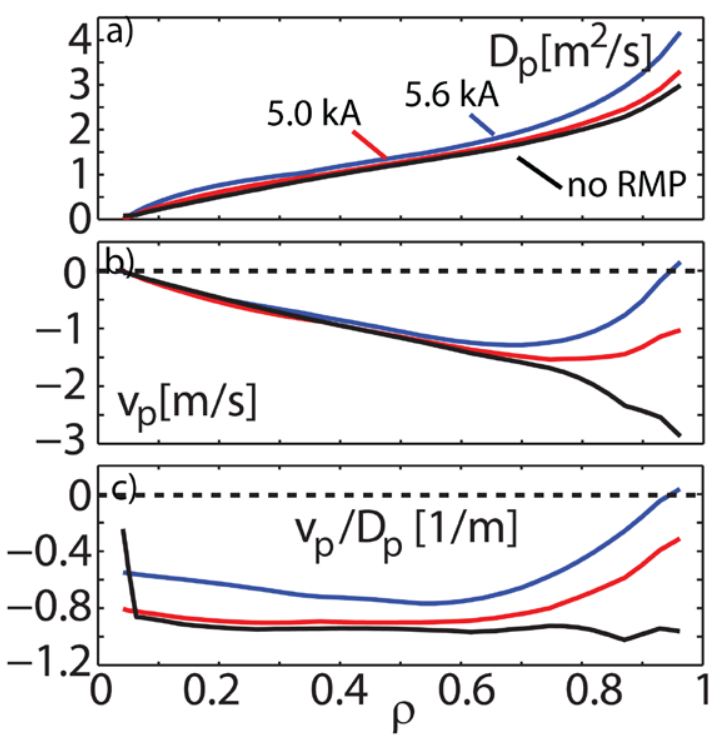

Figure 8. (a) The measured perturbed diffusion coefficient, $D_{p}$ and (b) particle pinch, $v_{p}$ and (c) the ration of $v_{p} / D_{p}$ for the three discharges from figure 3 using a small perturbative gas puff. The diffusion coefficient increases with increasing RMP amplitude, while the inward pinch mostly decreases at the plasma edge, to the point of reversing close to the seperatrix for the highest RMP coil current (blue). There is a clear change at the plasma edge of the $v_{p} / D_{p}$ ratio and for the discharge with the island, the change in transport is over the whole minor radius.

the island and the gradients increase just outside the island boundary and using the gas puff modulation technique also showed an increase in diffusion [27]. Future work will include an improvement of capturing the edge fueling to compare $v_{p} / D_{p}$ with the inverse density gradient along with including the steady state fueling source in the calculation of $v_{p}$ and $D_{p}$ [28]. This will allow us to assess whether the perturbed $v_{p} / D_{p}$ are in agreement with the steady state particle transport. 


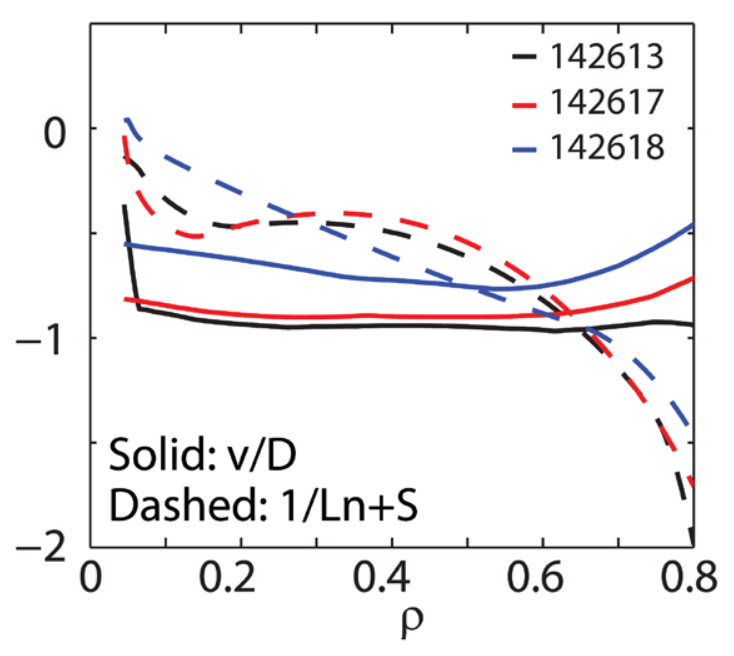

Figure 9. Comparison of $1 / L_{n}+\int S / D n$ (dashed) with $v_{p} / D_{p}$ (solid) for all three discharges from figure 3 .

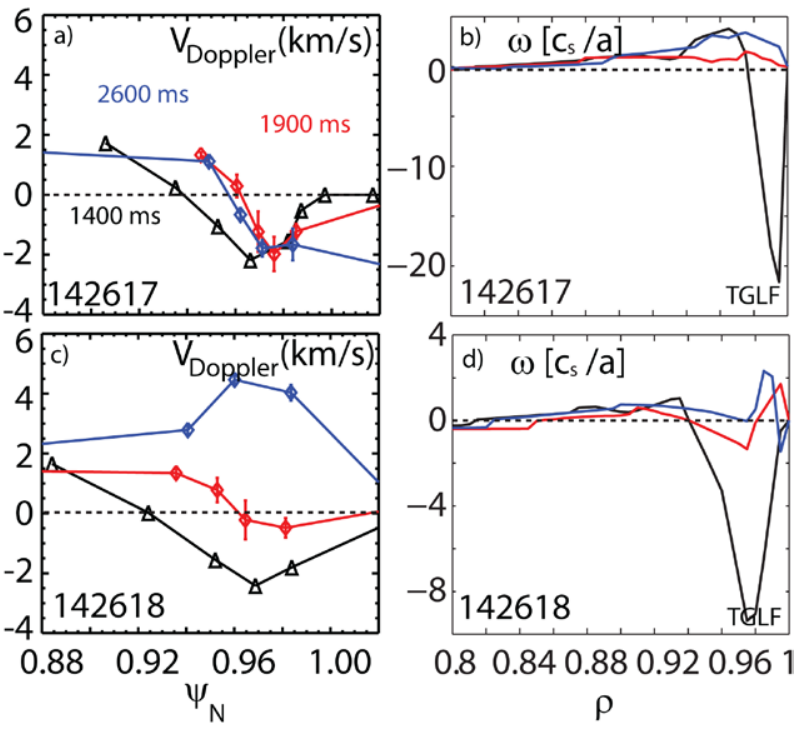

Figure 10. Comparison of the flow measured by the DBS system (a) and (c) with linear gyrokinetic simulations of the frequency at $k_{\theta} \rho_{s}=0.45$ (b) and (d) at three distinct times for both discharges in which RMPs are applied: $1400 \mathrm{~ms}$ (black) before the RMP coils are activated. $1900 \mathrm{~ms}$ (red) during the strong $n=3$ plasma response phase and at $2600 \mathrm{~ms}$ (blue), still during the plasma response phase for the 142617 , but after the 3D fields penetrate for 142618 .

\subsection{Turbulence}

In the previous section we show that the particle pinch changes from inward to outward when RMPs are applied at the plasma edge. In order to better understand what causes this change in transport we calculate the linear stability using TGLF [29]. We find that at the plasma edge, in the non-RMP discharge, the frequency of linear growth rates at $k_{\theta} \rho_{s}=0.45$ is in the ion direction, see figures $10(\mathrm{~b})$ and (d). Here $k_{\theta}$ is the wave number and $\rho_{s}$ is the ion gyro-radius. The frequency increases when RMPs are applied and switches to the electron direction close to the seperatrix by $2600 \mathrm{~ms}$ for both the $5.0 \mathrm{kA}$ and the $5.6 \mathrm{kA}$ discharges. Theoretically, when the turbulence regime changes from being dominantly ion temperature gradient

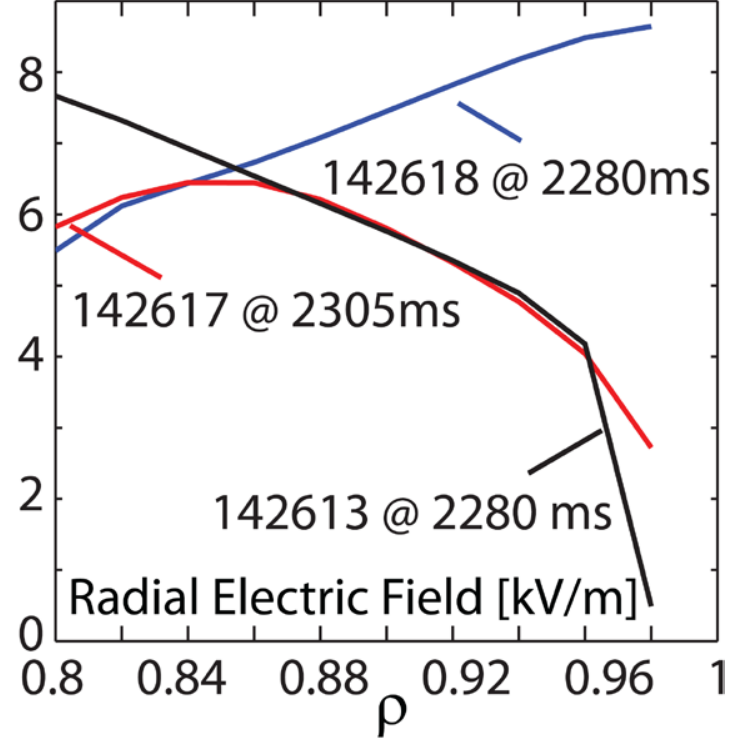

Figure 11. Edge radial electric field for all three discharges from figure 3. From $\rho \sim 0.86$ the no RMP discharge (black) and the 5.0 kA case (red) are very similar with exception at $\rho=0.98$. The 5.6 $\mathrm{kA}$ (blue) has a very different radial electric field. This increase is consistent with the changes in the edge rotation.

(ITG) unstable to being dominantly trapped electron mode (TEM) unstable, this results in the partial reversal of particle pinch $[18,19]$. The linear simulations from TGLF seem to be in good agreement with the observations in figure 8 . Figures 10(a) and (c) show the changes in flow measured with the Doppler backscattering (DBS) system [30]. Only when the 3D field penetrates, do we observe a change in direction of the DBS flow. The flow from the DBS is measured in the lab frame and we need to subtract the changes from the $E \times B$ flow in order to get the the changes in the turbulence flow.

From $\rho=0.86-0.94$ there is no change in the radial electric field, $E_{\mathrm{r}}$ for the discharge without RMPs and with $5.0 \mathrm{kA}$ discharge, see figure 11 . This means that any changes observed by the DBS at these locations, are the result of a change in turbulent flow. The discharge with 5.6 kA RMP coil current has very different $E_{\mathrm{r}}$. This change is dominated by the spin up in the toroidal rotation at the plasma edge, see figure 4(d). This change in $E_{\mathrm{r}}$ also dominates the observed reversal of the flow in figure $10(\mathrm{c})$.

It appears from the TGLF simulations and the flow measurements from the DBS that there is a transition in the plasma edge from being ITG dominant to becoming TEM dominant. In figure 12 we compare the changes in density fluctuations for the same discharges and times as figure 10 with the linear growth rates. We find that for the $5.0 \mathrm{kA}$ case, changes in density fluctuations as measured with the DBS, ranging from $k_{\theta} \rho_{s} \sim 0.2-0.45$ is in good agreement with the changes observed in the linear growth rates. For the $5.6 \mathrm{kA}$ discharge, the linear growth rates are not in agreement. One interesting observation is that during the plasma response phase at $1900 \mathrm{~ms}$ the $5.0 \mathrm{kA}$ and the $5.6 \mathrm{kA}$ discharge have very different fluctuation levels. The increase afterwards at $2600 \mathrm{~ms}$ for the $5.6 \mathrm{kA}$ discharge is also not observed in the linear growth rates. 

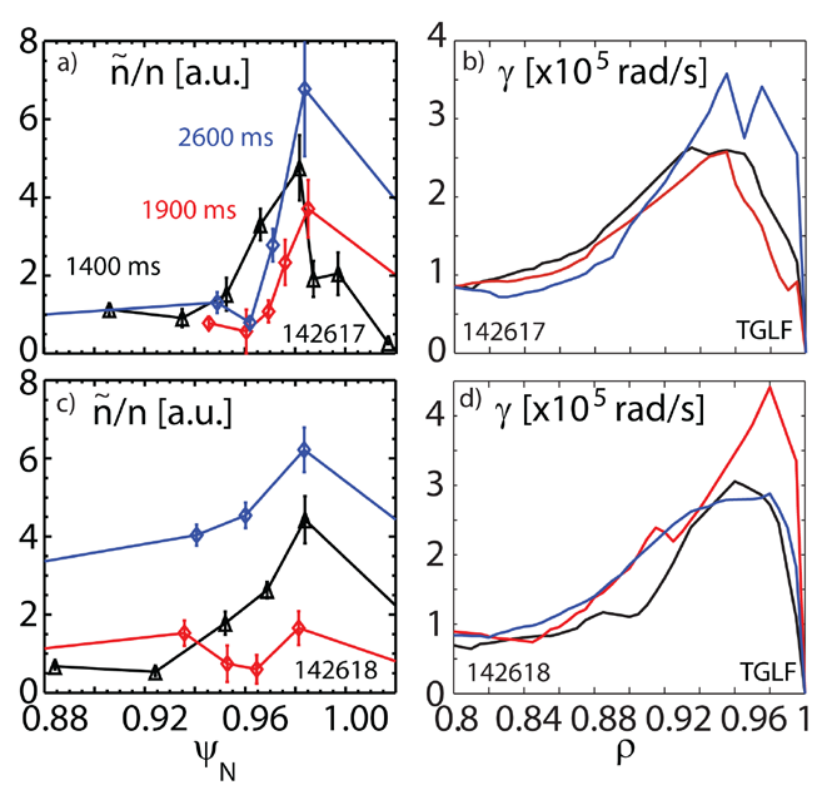

Figure 12. Comparison of the density fluctuations by the DBS system for $k_{\theta} \rho_{\mathrm{s}} \sim 0.2-0.45$ (a) and (c) with linear gyrokinetic simulations of the growth rate at $k_{\theta} \rho_{s}=0.45$ (b) and (d) at three distinct times for both discharges in which RMPs are applied: $1400 \mathrm{~ms}$ (black) before the RMP coils are activated. $1900 \mathrm{~ms}$ (red) during the strong $n=3$ plasma response phase and at $2600 \mathrm{~ms}$ (blue), still during the plasma response phase for the 142617 , but after the 3D fields penetrate for 142618 .

\section{Discussion}

When comparing the results presented in the previous section, we find that there are some differences with previous results as well as similarities. In comparison to the L-mode results from MAST, we do observe a small increase in $E_{\mathrm{r}}$, very close to the seperatrix, however the density fluctuations do not increase and the local density is not reduced [17]. The turbulence and transport changes with RMPs in these DIII-D plasmas, push the edge plasma across the ITG-TEM boundary, instead of simply increasing turbulent transport in the ITG regime.

It is more difficult to compare these results to the TEXTOR L-mode results. In TEXTOR, vacuum simulations were able to distinguish between different regions of 3D fields. In these DIII-D plasmas, considering the plasma response changes, we can not rely on vacuum simulations to approximate the $3 \mathrm{D}$ character of the plasma edge. What is interesting to observe is that the changes in profiles and turbulence that are on TEXTOR associated with the laminar zone are not observed in these plasmas [15]. However, the observations typical of the ergodic zone, such as no changes in electron temperature gradient, a steepening of the density, a reduction in large scale structures, are observed in the plasmas during which we measure an $n=3$ plasma response. In TEXTOR reversal of the particle flux is also observed in this region, but the flux changes from outward to inward, whereas our perturbed measurements indicate a change from inward to outward. We do not observe the creation of a $E_{\mathrm{r}}$ well along with a local flattening of $T_{\mathrm{e}}$ at the plasma edge [14]. There is some flattening of $T_{\mathrm{e}}$ once the plasma response disappears, which correlates with the creation of islands and is a local effect, not a global effect. In this case, when the field penetrates and the edge becomes stochastic, we observe that the radial electric field becomes positive as a result of the spin up in the toroidal rotation. This is similar to observations on TEXTOR, outside the separatrix.

These changes in turbulence and transport are in sharp contrast to the changes that are typically observed in DIII-D $\mathrm{H}$-mode plasmas. In H-mode, in the steep part of the pedestal, the changes in density fluctuations at different scales, correlate with the changes in the linear growth rates [25]. From the top of the pedestal and inward, the density fluctuations increase along with a strong decrease in the $E \times B$ shearing rate. The reduction in $E \times B$ is so large, that the linear growth rates become comparable to the shearing rate. This is an indication that the local shear becomes ineffective at suppressing turbulent transport and thus reducing particle confinement.

It is clear that all these changes in turbulence and transport at the plasma edge in L-mode will affect the L- to $\mathrm{H}$-mode transition. The changes in the local shearing rate is small in these L-mode plasmas, whereas in previous results, the changes in $E_{\mathrm{r}}$ and rotation were crucial in explaining the increase in the L- to H-mode power threshold [8-10]. In these DIII-D plasmas, we find that dominant change is a switch from the ITG to the TEM turbulence regime. How this change in turbulence regime and thus particle pinch will affect the access to H-mode is not yet understood.

\section{Conclusion}

In this paper we present the first experimental results of $3 \mathrm{D}$ fields in L-mode plasmas upon turbulence and particle transport studies on DIII-D. We find that at lower RMP currents, when we measure a strong $n=3$ plasma response, linear gyrokinetic simulations indicate that the plasma transitions from an ITG to a TEM dominant regime. Along with this change, we also observe a reversal of the particle pinch at the plasma edge. The DBS also shows that the flow becomes positive, while at the same radii no change in the radial electric field is observed. The change in turbulence characteristics will affect the L- to H-mode transition, even if the changes in the radial electric field and fluctuation levels are small. At higher RMP currents, the creation of a 3D stochastic edge, results in the spin up of the edge rotation. This spin up of the edge rotation, results in a positive radial electric field, along with an increase in density fluctuations. This increase in fluctuations is not captured by the 1D linear gyrokinetic simulations.

\section{Acknowledgments}

This material is based upon work supported by the U.S. Department of Energy, Office of Science, Office of Fusion Energy Sciences, using the DIII-D National Fusion Facility, a DOE Office of Science user facility, under Awards DE-SC0007880, DE-FG02-08ER54984, DE-FC0204ER54698, and DE-SC0001961. DIII-D data shown in this paper can be obtained in digital format by following the links at https://fusion.gat.com/global/D3D_DMP. We would like to 
especially thank P Peers as well as C Greenfield for advocating for run time for that opinionated graduate student who kept pestering him.

\section{References}

[1] Evans T et al 2006 Nat. Phys. 2 419-23

[2] Hawryluk R, Eidietis N, Grierson B, Hyatt A, Kolemen E, Logan N, Nazikian R, Paz-Soldan C, Solomon W and Wolfe S 2015 Nucl. Fusion 55053001

[3] Loarte A et al 2014 Nucl. Fusion 54033007

[4] Coenen J et al 2015 Nucl. Fusion 55023010

[5] Loarte A et al 2003 Plasma Phys. Control. Fusion 451549

[6] Zohm H 1996 Plasma Phys. Control. Fusion 38105

[7] Gohil P, Evans T, Fenstermacher M, Ferron J, Osborne T, Park J, Schmitz O, Scoville J and Unterberg E 2011 Nucl. Fusion $\mathbf{5 1} 103020$

[8] Ryter F, Rathgeber S, Viezzer E, Suttrop W, Burckhart A, Fischer R, Kurzan B, Potzel S, Ptterich T and the ASDEX Upgrade Team 2012 Nucl. Fusion 52114014

[9] Kaye S et al 2011 Nucl. Fusion 51113019

[10] Scannell R, Kirk A, Carr M, Hawke J, Henderson S, Gorman T, Patel A, Shaw A, Thornton A and the MAST Team 2015 Plasma Phys. Control. Fusion 57075013

[11] Schmitz L, Zeng L, Rhodes T L, Hillesheim J C, Doyle E J, Groebner R J, Peebles W A, Burrell K H and Wang G 2012 Phys. Rev. Lett. 108155002

[12] Devynck P et al 2002 Nucl. Fusion 42697

[13] Xu Y et al 2009 Nucl. Fusion 49035005

[14] Xu Y et al 2006 Phys. Rev. Lett. 97165003

[15] Xu Y et al 2007 Nucl. Fusion 471696
[16] Xu Y, Jachmich S, Van Schoor M, Vergote M, Jakubowski M W and Weynants R R 2007 J. Nucl. Mater. 363-365 718-22

[17] Tamain P, Kirk A, Nardon E, Dudson B, Hnat B and the MAST team 2010 Plasma Phys. Control. Fusion 52075017

[18] Garbet X, Garzotti L, Mantica P, Nordman H, Valovic M, Weisen H and Angioni C 2003 Phys. Rev. Lett. 91035001

[19] Angioni C, Camenen Y, Casson F, Fable E, McDermott R, Peeters A and Rice J 2012 Nucl. Fusion 52114003

[20] Ryter F, Orte L B, Kurzan B, McDermott R, Tardini G, Viezzer E, Bernert M, Fischer R and The ASDEX Upgrade Team 2014 Nucl. Fusion 54083003

[21] Mordijck S, Moyer R, Ferraro N, Wade M and Osborne T 2014 Nucl. Fusion 54082003

[22] Schmitz O, Evans T, Fenstermacher M, Lanctot M, Lasnier C, Mordijck S, Moyer R and Reimerdes H 2014 Nucl. Fusion 54012001

[23] Haskey S R, Lanctot M J, Liu Y Q, Paz-Soldan C, King J D, Blackwell B D and Schmitz O 2015 Plasma Phys. Control. Fusion 57025015

[24] Moyer R, Zeeland M V, Orlov D, Wingen A, Evans T, Ferraro N, Hanson J, Nazikian R, Wade M and Zeng L 2012 Nucl. Fusion $\mathbf{5 2} 123019$

[25] Mordijck S et al 2012 Phys. Plasmas 19056503

[26] Takenaga H, Nagashima K, Sakasai A, Oikawa T and Fujita T 1998 Plasma Phys. Control. Fusion 40183

[27] Jakubowski M et al 2013 Nucl. Fusion 53113012

[28] Salmi A et al 2014 Gas puff modulation experiments in jet L- and H-mode plasmas 40th EPS Conf. (http://iocs.ciemat. es/EPS2014PAP/pdf/Pl.008.pdf)

[29] Staebler G, Kinsey J and Waltz R 2007 Phys. Plasmas 14055909

[30] Hillesheim J C, Peebles W A, Rhodes T L, Schmitz L, Carter T A, Gourdain P A and Wang G 2009 Rev. Sci. Instrum. 80083507 